Patricia Farías ${ }^{1}$

Sonia Tifner ${ }^{2}$ María Lourdes Tapia ${ }^{3}$

${ }^{1}$ Trabaja en Clínica Los Álamos. San Juan. E-mail: paedfa@hotmail.com ${ }^{2}$ Prof. Responsable cátedra Psicopatología Cognitivo Integrativo. Facultad de Psicología. UNSL. PROICO:

12/0314, UNSL. E-mail: stifner@unsl.edu.ar ${ }^{3}$ JTP Exclusiva efectiva de la cátedra Modelos Cognitivo Conductuales. Facultad de Psicología, UNSL. PROICO 12/0316. E-mail: mtapia@ unsl.edu.ar

Trabajo recibido: 27 de Junio de 2017.

Aprobado: 04 de Noviembre de 2017.

\section{EVALUACIÓN DE LA RELACIÓN MÉDICO PACIENTE (RMP) EN UNA POBLACIÓN DE SAN JUAN.}

ASSESSMENT OF DOCTOR-PATIENT RELATIONSHIP (DPR) IN A POPULATION IN SAN JUAN.

AVALIAÇÃO DO RELACIONAMENTO MÉDICO-PACIENTE (RMP) EM UMA LOCALIDADE DE SAN JUAN.

\section{Resumen}

El objetivo del trabajo fue indagar la calidad de la Relación Médico Paciente(RMP) en pacientes de una localidad de la provincia de San Juan. Se realizó una encuesta a usuarios de una clínica privada y hospital público sobre la calidad de la relación médico paciente. Resultados: Más del $70 \%$ de los encuestados refirió que su médico lo entiende, que confía en él, que se esfuerza en ayudarlo, que puede hablar él, que está contento con el tratamiento que le ha puesto, que le ayuda, que el tratamiento lo está beneficiando, que está de acuerdo con éste sobre las causas de sus síntomas, que es muy accesible, refieren sentirse mejor gracias a su médico, considera que tiene más información sobre su salud debido a su médico, sostiene que ahora controla mejor los síntomas aunque no vea a su médico. Los respondientes de la clínica privada perciben que pueden hablar más con su médico y que lo encuentran más accesible que los usuarios del hospital público. Discusión: En todos los ítems del cuestionario más del 50\% de los encuestados refiere una buena RMP.

Palabras clave: Relación, médico, paciente, hospital, público, privado. 


\section{Abstract}

Abstract: The objective of this study was to inquire about the quality of Doctor-Patient Relationship (DPR) in patients in a town in the province of San Juan. A survey to patients about doctor-patient relationship was carried out in a private clinic and in a private hospital. Results: More than $70 \%$ of the contestants said they trust their doctor, their doctor see to them, helps them, makes an effort to help them, is approachable, it is possible to talk to, that they are happy with the prescribed treatment, that the treatment is helping them and it is beneficial to them, that they agree with the doctor on the causes of symptoms, and they say they feel better thanks to their doctor and consider they have more information about their health because of their doctor and that they have a better control of the symptoms even when they do not see the doctor. Contestants from the private clinic perceive that they can talk more to the doctor and find doctors more approachable than users of the public hospital. Discussion: In all the items of the questionnaire, more than $50 \%$ of patients refer to a good DPR.

Key words: Relationship, doctor, patient, hospital, public, private.

\section{Resumo}

O alvo do trabalho foi pesquisar a qualidade da Relação Médico- Paciente (RMP) em pacientes de uma localidade da província de San Juan. Foi realizada uma enquete para usuários de uma clínica privada e de um hospital público sobre a qualidade do relacionamento do paciente com o médico. Resultados: Mais de $70 \%$ dos entrevistados relataram que seu médico os entendia, que confiavam nele, que ele fazia um esforço para ajudá-los, que eles podiam falar, que eles estavam felizes com o tratamento que ele lhes dava, que ele os ajudava, que estavam se beneficiando com o tratamento, que eles concordavam com ele sobre as causas de seus sintomas, que ele era muito acessível, eles relatam sentir-se melhor graças ao seu médico, eles consideram que ele tem mais informações sobre sua saúde devido ao seu médico, ele afirma que ele agora controla melhor os sintomas mesmo se não vê seu médico. Os entrevistados da clínica privada percebem que podem conversar mais com seu médico e que acham isso mais acessível do que os usuários do hospital público. Discussão: Em todos os itens do questionário, mais de $50 \%$ dos entrevistados relataram um bom RMP.

Palavras chave: Relacionamento, médico, paciente, hospital, público, privativo.

\section{Introducción}

Dentro de las relaciones humanas, la Relación Médico Paciente (RMP) es una de las más complejas e intensas. Esta relación está matizada y en ocasiones determinada por el contexto histórico, cultural, social y económico en el que el saber y la práctica médica se insertan y está supeditada a factores humanos (1).

Díaz Muñoz (2) sostiene que la relación médico-paciente, es la base fundamental de una buena consulta médica, tiene connotaciones humanas, éticas, filosóficas, sociológicas y hasta implicaciones legales por las consecuencias que su alteración puede producir. Debe ser considerada por los profesionales como un pilar esencial del ejercicio médico y los pacientes deben reclamarlo como un derecho.

La RMP no habrá de ser simétrica ni armónica, sino que habrá de caracterizarse por la coexistencia de encuentros y desencuentros entre el paciente y el médico, como consecuencia de las diferentes subjetividades que se ponen en juego en la relación. Por ello cada relación médico paciente conformará una original y particular modalidad de vínculo único e irrepetible (3). 
La relación médico-paciente no debe ser sólo una variable a tener en cuenta en el acto clínico, sino que debe tener un lugar central y debe ser uno de los principales instrumentos del médico. Se debe procurar que el médico sea cada vez mejor profesional y que sus habilidades no se vean disminuidas por la dureza de las tareas que debe desempeñar, como es el enfrentarse a diario al dolor, a las angustias y sufrimiento ajeno (2).

Por todo lo expresado, el objetivo del presente trabajo de investigación es indagar la calidad de la RMP en pacientes de una localidad de la provincia de San Juan.

A partir de conocer esta experiencia de los pacientes se podría aportar al conocimiento de los aspectos del vínculo que podrían afectar por ejemplo a la adherencia al tratamiento para futuras investigaciones

\section{Metodología}

Esta investigación es descriptiva, exploratoria y transversal.

Participantes: 100 pacientes, mujeres y varones, de una localidad de San Juan,

Instrumentos:

1- Cuestionario de Relaciones Médico-Paciente (CREM-P) de Mingote Adán, MorenoJiménez, Rodríguez Carvajal, Gálvez Herrer y Ruiz-López (4); que evalúa la calidad de la relación médico-paciente. El mismo consta de 13 ítems y se muestra como un instrumento fiable, válido y de fácil cumplimentación, diseñado para permitir la evaluación tanto en contextos clínicos como de investigación..

Los autores procedieron a la adaptación al castellano del cuestionario PDQR (PatientDoctor Relation ship Questionnaire) de Van der Feliz-Cornelis, Van Oppen, Van Marwijk, De Beurs y Van Dyck, 2004 (5) en su versión de 15 ítems. En primer lugar realizaron la traducción del cuestionario al español (CREM-P) y posteriormente lo retradujeron al inglés, evaluando el nivel de precisión y ajuste de las diferencias encontradas.

El cuestionario presenta una escala de tipo Likert de 6 puntos donde 1 significa totalmente en desacuerdo, 2 en desacuerdo, 3 levemente en desacuerdo, 4 levemente de acuerdo, 5 de acuerdo y 6 totalmente de acuerdo. Las puntuaciones implican que a mayor puntaje mayor acuerdo, en tanto que a menor puntaje mayor desacuerdo.

Procedimiento: Se fue a las salas de espera del Hospital Público y Sanatorios privados donde, previo consentimiento de los pacientes, se les administró los instrumentos de forma individual.

\section{Resultados}

Fueron 100 los usuarios de los servicios de hospital público y clínica privada, (50 de ámbito de salud pública y 50 de ámbitos privados). Teniendo en cuenta el género, el 62 $\%$ de los entrevistados pertenecían al sexo femenino y $38 \%$ al sexo masculino. Respecto a las edades, la máxima fue de 86 años y la mínima de 18, siendo la edad promedio de 36 años. En lo que atañe a nivel de escolaridad: el $59 \%$ cursó el secundario, el $20 \%$ el nivel primario, el $11 \%$ accedió a un grado universitario y el $8 \%$ a un nivel terciario.

En cuanto al estado civil, el $55 \%$ de los entrevistados eran solteros, el $36 \%$ casados, un 5 $\%$ manifestó ser viudo y el $4 \%$ restante indicó ser divorciado. El $62 \%$ refirió tener hijos, frente a un $38 \%$ que dijo no tenerlos.

Teniendo en cuenta toda la muestra, es decir, los usuarios de la clínica privada como del hospital público, se describen las medias como los desvíos estándar de cada ítem del cuestionario de la relación médico paciente (CREM-P). 
Tabla $\mathbf{N}^{0}$ 1: Estadísticos descriptivos de cada ítem del CREM P

\begin{tabular}{|c|c|c|c|c|c|}
\hline & $\mathbf{N}$ & Mínimo & Máximo & Media & $\begin{array}{l}\text { Desviación } \\
\text { estándar. }\end{array}$ \\
\hline $\begin{array}{l}\text { Mi médico me } \\
\text { ayuda }\end{array}$ & 100 & 1,00 & 6,00 & 4,8500 & 1,29002 \\
\hline $\begin{array}{l}\text { Mi médico me } \\
\text { dedica suficiente } \\
\text { tiempo } \\
\text { El tratamiento que }\end{array}$ & 100 & 1,00 & 6,00 & 4,3800 & 1,50272 \\
\hline $\begin{array}{l}\text { me ha puesto mi } \\
\text { médico me está } \\
\text { beneficiando } \\
\text { Estoy de acuerdo }\end{array}$ & 100 & 1,00 & 6,00 & 4,9900 & 1,13258 \\
\hline $\begin{array}{l}\text { con mi médico } \\
\text { sobre la causa de } \\
\text { mis síntomas }\end{array}$ & 100 & 1,00 & 6,00 & 4,9200 & 1,08879 \\
\hline $\begin{array}{l}\text { Encuentro a mi } \\
\text { médico muy } \\
\text { accesible }\end{array}$ & 100 & 1,00 & 6,00 & 4,7600 & 1,37157 \\
\hline $\begin{array}{l}\text { Gracias a mi } \\
\text { médico me } \\
\text { encuentro mejor }\end{array}$ & 100 & 1,00 & 6,00 & 5,0500 & 1,20918 \\
\hline $\begin{array}{l}\text { Gracias a mi } \\
\text { médico tengo más } \\
\text { información acerca } \\
\text { de mi salud } \\
\text { Ahora controlo } \\
\text { mejor a mis }\end{array}$ & 100 & 1,00 & 6,00 & 4,9200 & 1,29240 \\
\hline $\begin{array}{l}\text { síntomas (aunque } \\
\text { vea menos al } \\
\text { médico) }\end{array}$ & 100 & 1,00 & 6,00 & 4,8900 & 1,14499 \\
\hline $\begin{array}{l}\mathrm{N} \text { válido (según } \\
\text { lista) }\end{array}$ & 100 & & & & \\
\hline
\end{tabular}

Teniendo en cuenta que a partir de la puntuación $n^{\circ} 4$ del cuestionario (levemente de acuerdo), se puede decir, observando las medias de la tabla $\mathrm{n}^{\circ}$ 1que hay promedios de acuerdo en todos los ítems. Dato éste coincidente con los mayores porcentajes de acuerdo reflejados en la tabla $n^{\circ} 2$.

En la tabla 2 se describe la información más relevante (mayores porcentajes) respecto de cada ítem.

Tabla $\mathbf{N}^{0}$ 2: Mayores porcentajes de respuesta de cada ítem de la encuesta CREM- P

\begin{tabular}{|c|c|c|c|}
\hline CREMP & $\begin{array}{c}\text { Acuerdo } \\
\text { desacuerdo }\end{array}$ & Frecuencia & Porcentaje \\
\hline Ítem No 1: Mi médico me entiende & De acuerdo & 41 & $410 \%$ \\
\hline Ítem No 2: Confío en mi médico & De acuerdo & 42 & $42,0 \%$ \\
\hline Ítem N³: Mi médico se esfuerza en ayudarme & De acuerdo & 38 & $38,0 \%$ \\
\hline Ítem No 4: Puedo hablar con mi médico & $\begin{array}{c}\text { Totalmente de } \\
\text { acuerdo }\end{array}$ & 47 & $47,0 \%$ \\
\hline $\begin{array}{c}\text { Ítem } N^{\circ} \text { 5: Estoy contento con el tratamiento que } \\
\text { me ha puesto mi médico }\end{array}$ & \begin{tabular}{c} 
De acuerdo \\
\hline
\end{tabular} & 40 & $40,0 \%$ \\
\hline
\end{tabular}




\begin{tabular}{|c|c|c|c|}
\hline Ítem $N^{\circ} 6$ : Mi médico me ayuda & De acuerdo & 40 & $40,0 \%$ \\
\hline $\begin{array}{c}\text { Ítem } N^{\circ} 7: \text { Mi médico me dedica suficiente } \\
\text { tiempo }\end{array}$ & $\begin{array}{l}\text { Totalmente de } \\
\text { acuerdo }\end{array}$ & 29 & $29,0 \%$ \\
\hline $\begin{array}{l}\text { Ítem N 8: El tratamiento que me ha puesto mi } \\
\text { médico me está beneficiando }\end{array}$ & De acuerdo & 42 & $42,0 \%$ \\
\hline $\begin{array}{c}\text { Ítem } N^{\circ} \text { 9: Estoy de acuerdo con la causa de } \\
\text { mis síntomas }\end{array}$ & De acuerdo & 49 & $49,0 \%$ \\
\hline $\begin{array}{c}\text { Ítem N 10: Encuentro a mi médico muy } \\
\text { accesible }\end{array}$ & $\begin{array}{l}\text { Totalmente de } \\
\text { acuerdo }\end{array}$ & 36 & $36,0 \%$ \\
\hline $\begin{array}{c}\text { Ítem No 11: Gracias a mi médico me encuentro } \\
\text { mejor }\end{array}$ & $\begin{array}{l}\text { Totalmente de } \\
\text { acuerdo }\end{array}$ & 46 & $46,0 \%$ \\
\hline $\begin{array}{c}\text { Ítem N } 12: \text { Gracias a mi médico tengo más } \\
\text { información sobre mi salud }\end{array}$ & $\begin{array}{l}\text { Totalmente de } \\
\text { acuerdo }\end{array}$ & 43 & $43,0 \%$ \\
\hline $\begin{array}{c}\text { Ítem } N^{\circ} \text { 12: Ahora controlo mejor mis síntomas } \\
\text { (aunque vea menos al médico) }\end{array}$ & De acuerdo & 45 & $45,0 \%$ \\
\hline
\end{tabular}

Con respecto a la primera afirmación "Mi médico me entiende"(ítem $\mathrm{N}^{\circ}$ 1) el mayor porcentaje $(41 \%)$ respondió estar de acuerdo.

En la aseveración "Confío en mi médico"(ítem $\mathrm{N}^{\circ} 2$ ), los porcentajes más elevados reflejan que los pacientes depositan su confianza en el médico (el $42 \%$ dijo estar de acuerdo).

En la consigna "Mi médico se esfuerza en ayudarme" (ítem $N^{\circ} 3$ ) se observa que la mayoría de las personas encuestadas manifestaron estar de acuerdo con esta afirmación (38\%).

En la afirmación "Puedo hablar con mi médico"(ítem $\mathrm{N}^{\circ} 4$ ), los porcentajes más altos se ubicaron en respuestas positivas respecto a la comunicación paciente-médico, refiriendo el $47 \%$ de los sujetos, estar totalmente de acuerdo.

En relación a la consigna "Estoy contento con el tratamiento que me ha puesto mi médico" (ítem $\mathrm{N}^{\circ}$ 5): el $40 \%$ de los entrevistados dijo estar de acuerdo. .

Respecto a la afirmación que expresa "Mi médico me ayuda" (ítem $\mathrm{N}^{\circ} 6$ ), la mayoría de los entrevistados (40\%) indicó estar de acuerdo en recibir ayuda por parte del médico que los atiende.

El $29 \%$ de los encuestados reveló estar totalmente de acuerdo respecto a la enunciación "Mi médico me dedica suficiente tiempo" (ítem $\mathrm{N}^{\circ} 7$ ).

Acerca de "El tratamiento que me ha puesto mi médico me está beneficiando" (ítem № 8), el mayor porcentaje de los usuarios alegó estar de acuerdo (42\%).

El $49 \%$ de las personas entrevistadas dijo estar de acuerdo con el médico, respecto a las causas de la sintomatología manifestada (ítem $\mathrm{N}^{\circ}$ 9).

La mayoría de los encuestados manifestó estar totalmente de acuerdo (36\%) con esta consigna, encontrando a su médico accesible y próximo cuando se lo requiera (ítem $\mathrm{N}^{\circ}$ $10)$.

En relación a esta afirmación, un gran porcentaje de sujetos (46 \% ) atribuyen a su médico la mejoría en su salud (ítem $\left.\mathrm{N}^{\circ} 11\right)$.

Un elevado porcentaje de sujetos (43\% están totalmente de acuerdo), que la fuente más importante de información sobre aspectos asociados a la salud, proviene de su médico (item $\mathrm{N}^{\circ} 12$ ).

En lo que respecta a la última afirmación (ítem $\mathrm{N}^{\circ} 13$ ), se advierte que el mayor porcentaje de personas (45\% están de acuerdo) actualmente sienten controlar los síntomas de su enfermedad independientemente del número de visitas al médico. 
Tabla N³: Comparación de la calidad de la relación médico paciente en un hospital público y una clínica privada evaluada por los usuarios de los servicios

\begin{tabular}{|c|c|c|c|c|c|}
\hline Ítems CREM-P & $\begin{array}{l}\text { Tipo de } \\
\text { atención } \\
\text { médica }\end{array}$ & $\mathbf{N}$ & Media & $\begin{array}{l}\text { Desviación } \\
\text { estándar }\end{array}$ & $\begin{array}{c}\mathbf{p} \\
\text { (significación) }\end{array}$ \\
\hline \multirow{2}{*}{$\begin{array}{l}\text { Mi médico me } \\
\text { entiende }\end{array}$} & Pública & 50 & 4,8600 & 1,27791 & \\
\hline & Privada & 50 & 5,0400 & 87970 & 414 \\
\hline \multirow{2}{*}{$\begin{array}{l}\text { Mi médico se } \\
\text { esfuerza en } \\
\text { ayudarme }\end{array}$} & Pública & 50 & 4,7400 & 1,33722 & \\
\hline & Privada & 50 & 5,0600 & ,91272 & , 165 \\
\hline \multirow{2}{*}{$\begin{array}{l}\text { Puedo hablar con } \\
\text { mi médico }\end{array}$} & Pública & 50 & 4,8600 & 1,37039 & \\
\hline & Privada & 50 & 5,3600 & ,85141 & ,031 \\
\hline \multirow{3}{*}{$\begin{array}{l}\text { Estoy contento con } \\
\text { el tratamiento que } \\
\text { me ha puesto mi } \\
\text { médico }\end{array}$} & Pública & & & & \\
\hline & & 50 & 4,7800 & 1,26636 & \\
\hline & Privada & 50 & 5,0000 & ,92582 & ,324 \\
\hline \multirow{2}{*}{$\begin{array}{l}\text { Mi médico me } \\
\text { ayuda }\end{array}$} & Pública & 50 & 4,6200 & 1,45532 & \\
\hline & Privada & 50 & 5,0800 & 1,06599 &, 074 \\
\hline \multirow{2}{*}{$\begin{array}{l}\text { Mi médico me } \\
\text { dedica suficiente } \\
\text { tiempo }\end{array}$} & Pública & 50 & 4,1800 & 1,63744 & \\
\hline & Privada & 50 & 4,5800 & 1,34149 & , 185 \\
\hline \multirow{3}{*}{$\begin{array}{l}\text { El tratamiento que } \\
\text { me ha puesto mi } \\
\text { médico me está } \\
\text { beneficiando }\end{array}$} & Pública & & & & \\
\hline & & 50 & 4,9000 & 1,31320 & \\
\hline & Privada & 50 & 5,0800 & ,92229 & ,430 \\
\hline \multirow{3}{*}{$\begin{array}{l}\text { Estoy de acuerdo } \\
\text { con mi médico } \\
\text { sobre la causa de } \\
\text { mis síntomas }\end{array}$} & Pública & & & & \\
\hline & & 50 & 4,9200 & 1,20949 & \\
\hline & Privada & 50 & 4,9200 & ,96553 & 1,000 \\
\hline \multirow[t]{2}{*}{$\begin{array}{l}\text { Encuentro a mi } \\
\text { médico muy } \\
\text { accesible }\end{array}$} & Pública & 50 & 4,4600 & 1,50116 & \\
\hline & Privada & 50 & 5,0600 & 1,16776 & ,028 \\
\hline \multirow{2}{*}{$\begin{array}{l}\text { Gracias a mi } \\
\text { médico me } \\
\text { encuentro mejor }\end{array}$} & Pública & 50 & 4,8800 & 1,42342 & \\
\hline & Privada & 50 & 5,2200 & ,93219 & , 161 \\
\hline \multirow{3}{*}{$\begin{array}{l}\text { Gracias a mi } \\
\text { médico tengo más } \\
\text { información acerca } \\
\text { de mi salud }\end{array}$} & Pública & & & & \\
\hline & & 50 & 4,8000 & 1,45686 & \\
\hline & Privada & 50 & 5,0400 & 1,10583 & ,356 \\
\hline \multirow{3}{*}{$\begin{array}{l}\text { Ahora controlo } \\
\text { mejor a mis } \\
\text { síntomas (aunque } \\
\text { vea menos al } \\
\text { médico) }\end{array}$} & Pública & & & & \\
\hline & & 50 & 4,9600 & 1,15987 & \\
\hline & Privada & 50 & 4,8200 & 1,13731 & ,544 \\
\hline
\end{tabular}


Se observaron diferencias significativas en el ítem "Puedo hablar con mi médico" entre los usuarios de la clínica privada y el hospital público, obteniendo mayores puntuaciones los usuarios de la primera (p:,031).

En el ítem "Encuentro a mi médico muy accesible" los usuarios de la clínica privada obtuvieron mayores puntuaciones (diferencias significativas) p:,028 que las del hospital público.

Por otra parte, se observó una tendencia entre los usuarios de la clínica privada y el hospital público en el ítem "Mi médico me ayuda" observándose mayores puntuaciones en los usuarios de la entidad privada, diferencia no significativa, (p:,074).

\section{Discusión}

Se observó que la mayoría de los sujetos refieren recibir ayuda por parte del médico. Así mismo, la mayoría de los encuestados alegó estar más informado en lo que respecta a su salud, gracias al médico. Esto no concuerda con los resultados obtenidos por Barca Fernández, Parejo Miguez, Gutiérrez Martín, Fernández Alarcón, Alejandro Lázaro y López de Castro (6), quienes realizaron un trabajo cuyo objetivo era evaluar la información brindada por el médico en consulta, la solicitada por el paciente y su participación en la toma de decisiones.

Los autores estiman que la información proporcionada al paciente por el profesional no es todo lo completa que debería, sobre todo en lo referido a algunos aspectos concretos como sus posibles causas, el pronóstico y las posibles complicaciones del tratamiento. La información sobre el diagnóstico y el tratamiento se suministra con más frecuencia, aunque también es susceptible de mejorar, posiblemente porque se considera más básica. Los autores consideran que la participación del paciente en la toma de decisiones sigue siendo insuficiente a pesar de que parece probado que participar resulta positivo desde el punto de vista de la efectividad del tratamiento.

En relación al tiempo que le dedica el médico, el mayor porcentaje lo considera suficiente. Outomuroy Actis (7) plantean que la decisión acerca de cuánto tiempo el profesional de la salud estará cara a cara con el paciente resulta vital no sólo para programar la agenda de turnos sino también para garantizar la calidad de atención médica. Un turno demasiado corto ha sido descripto, entre otras cosas, como uno de los factores que impide la realización de una buena promoción de la salud. También es importante analizar la cantidad de tiempo, versus la calidad de tiempo. Esto implica una optimización del tiempo dedicado a la consulta médica evitando interrupciones que afectan la atención dedicada al enfermo, preguntas sin relevancia y desviaciones de la conversación hacia temas no vinculados con el problema médico. Varios estudios (8) sugieren que las consultas deberían tener una duración relativa de 20 min para satisfacer a los usuarios y cumplir con los estándares de calidad asistencial. Una encuesta realizada en México (9) entre los usuarios de los consultorios externos de varios centros asistenciales públicos y privados mostró que «para los usuarios de la seguridad social, los principales elementos que definen la mala calidad de atención son los largos tiempos de espera, los tiempos cortos dedicados a la consulta y la deficiencia en las acciones de revisión y diagnóstico, mientras que para la población abierta dichos elementos están constituidos por el tiempo corto que duran las consultas, las consultas diferidas y el mal trato por el personal que presta los servicios». En otro estudio semejante, (10) los principales factores asociados a una percepción general de baja calidad en la atención de las instituciones de salud fueron: el tipo de institución (público o privado), el tiempo de espera para acceder a la consulta, el grado de mejoría del estado de salud luego de la consulta y una duración de la consulta inferior a 20 minutos. En relación al tratamiento puesto por el médico, la mayoría de los entrevistados alegó que lo benefician. Badia y Lizán (11) mencionan que es muy importante la satisfacción con el tratamiento ya que con esta medida pueden conocerse los aspectos relacionados con el 
tratamiento que preocupan más al paciente. Esto permite mejorar el tratamiento presente y considerar dichos aspectos para aplicarlos en el futuro. El sentirse beneficiado es un factor importante a la hora de escoger un determinado tratamiento (incluido no tratar). En otros casos, la eficacia está disminuida, pero se ve compensada con una mayor comodidad de uso o falta de efectos adversos (12).La satisfacción con un tratamiento puede aumentar la adherencia. Si un paciente no está satisfecho con el modo de administración de un tratamiento o con su beneficio, es menos probable que cumpla con el régimen prescrito. Es posible que el hecho de conocer el grado de satisfacción del paciente con su tratamiento pueda contribuir a predecir el cumplimiento del paciente y ayudar al profesional en la toma de decisiones (13).

Los respondientes de la clínica privada perciben que pueden hablar más con su médico y que lo encuentran más accesible que los usuarios del hospital público. Quizás esto se deba a que los usuarios de hospitales públicos tienen largos períodos de espera y tiempos cortos dedicados a la consulta (Ortiz-Espinosa, Muñoz-Juárez, Martín del Campo y TorresCarreño, 2003) (9) en comparación con los de las clínicas privadas.

\section{Bibliografía}

1. Rodríguez Arce, M. A. Relación Médico-Paciente. La Habana: Editorial Ciencias Médicas; 2008.

2. 2: Díaz Muñoz, K. Relación entre la satisfacción del usuario y la calidad de la comunicación médico-paciente en el Centro de Salud de Conocoto. (Tesis para optar por el título de Diplomado de Gerencia y Calidad en servicios de Salud). Quito, Ecuador. Universidad Técnica Particular de Loja; 2011.

3. Artacho, J.. Relación médico paciente y subjetividad. Guía de Aprendizaje Crecimiento y Desarrollo. 2013; 1(10): 641-48.

4. Mingote Adán, J.C., Moreno-Jiménez, B., Rodríguez-Carvajal, R., Gálvez Herrer, M., Ruíz López, P. Validación psicométrica de la versión española del Cuestionario de Relaciones Médico-Paciente (CREM-P). Actas Españolas de Psiquiatría. 2008; 36:0-00.

5. Van der Feliz-Cornelis, C.M., Van Oppen, P., Van Marwijk, H.W.J., De Beurs, E. y Van Dyck, R. (2004). A patient-doctor relationshipquestionnaire (PDRQ-9) in primarycare: development and psychometricevaluation. Gen HospPsychiatry. 2004; 26:115-20.

6. Barca Fernández, I., Parejo Miguez, R., Gutiérrez Martín, P., Fernández Alarcón,F., Alejandre Lázaro, G. y López de Castro, F. La información al paciente y su participación en la toma de decisiones clínicas.Aten Primaria. 2003; 33 (7):361-7.

7. Outomuro, D. y Actis, A. M..Estimación del tiempo de consulta ambulatoria en clínica médica. Rev. méd. Chile, 141, No 3.[revista en internet]2013. Marzo. [acceso 2 de Mayo de 2017].Disponibleen:http://dx.doi.org/10.4067/S003498872013000300012

8. Lin, C. T., Albertson, G. A., Schilling, L. M., Cyran, E. M., Anderson, S. N. y Ware, L. Is patients> perception of time spent with the physician a determinant of ambulatory patient satisfaction?ArchInternMed.2001; 16: 1437-42.

9. Ortiz Espinosa, R. M., Muñoz Juárez, S., Martín del Campo, D. L. y Torres Carreño, E. Consulta externa en instituciones de Salud de Hidalgo, México, según la opinión de los usuarios. Pan Am J Public Health. 2003; 13(4): 229-38.

10. Sauceda Valenzuela, A.L., Wirtz, V. J., Santa Ana Tellez, Y., y Kageyama Escobar, M. L. Ambulatory health service users〉 experience of waiting time and expenditure and factors associated with the perception of low quality of care in México. BMC HealthServicesResearch. 2010; 10: 178-188.

11. Badia, X. y Lizán L. Reflexiones sobre la investigación de resultados en salud. Aten 
Primaria. 2002; 30: 388-91.

12. Herdman M. y Badia X. La medida de la satisfacción con el tratamiento en la Investigación de Resultados en Salud. En: Badia X. La investigación en resultados en salud.Barcelona: Edimac; 2000. p. 79-93.

13. Ruiz, M.A., Pardo, A., Rejas, J., Soto. J., Villasante, F. y Aranguren, J.L. Development and Validation of the "Treatment Satisfaction with Medicines Questionnaire".ValueHealth.2008; 11:913-26. 